\title{
Characterization and Monte Carlo simulations for a CLYC detector
}

\author{
Alessandro Borella, ${ }^{*}$ Eric Boogers ${ }^{\dagger}$ and Riccardo Rossa ${ }^{\ddagger}$ \\ $S C K \cdot C E N$, Belgian Nuclear Research Centre, \\ Boeretang 200, Mol, B2400, Belgium \\ *aborella@sckcen.be, ${ }^{\dagger}$ eboogers@sckcen.be, ${ }^{\dagger}$ rrossa@sckcen.be \\ Peter Schillebeeckx \\ European Commission, Joint Research Centre \\ Retieseweg 111, Geel, B2440, Belgium \\ peter.schillebeeckx@ec.europa.eu
}

Published 10 July 2018

\begin{abstract}
The CLYC $\left(\mathrm{Cs}_{2} \mathrm{LiYCl}_{6}: \mathrm{Ce}\right)$ detector is a scintillator detector sensitive to both neutron and gamma radiation and capable of separating the two types of radiation by pulse-shape discrimination. This feature is interesting as pertains to the development of non-destructive assays for the safeguard of nuclear material, nuclear security, and fast-neutron personal dosimetry. A 1" $\times 1$ " CLYC detector highly enriched with ${ }^{6} \mathrm{Li}$ was purchased and tested with analog and digital electronics. In this work, we report on the characterization of the detector in terms of linearity, energy resolution, and full-energy efficiency for gamma rays. This characterization was achieved by measurements with calibrated gamma-ray point-sources with an analog measuring chain, in a well-defined, reproducible geometry. The experimental data were also used to validate a model of the detection system that was developed with the Monte Carlo code MCNP-CP. This work is part of a collaborative agreement between SCK $\cdot \mathrm{CEN}$ and JRC-Geel.
\end{abstract}

Keywords: CLYC; Monte Carlo modeling; detector response; nondestructive assay; gamma-ray spectrometry; safeguards.

PACS numbers: 07.05.Tp, 07.85.Fv, 29.30.Kv, 29.40.-n, 29.40.Wk

\section{Introduction}

CLYC $\left(\mathrm{Cs}_{2} \mathrm{LiYCl}_{6}: \mathrm{Ce}\right)$ detector ${ }^{1}$ research and development has been presented in a number of papers. ${ }^{2-4}$ CLYC detectors work for both gamma rays and neutrons. Gamma radiation is detected with a typical energy resolution of less than $5 \%$ at $662 \mathrm{keV}$ and high linearity, ${ }^{4}$ and neutrons produce an observable signal following $(n, \alpha)$ reaction on ${ }^{6} \mathrm{Li}$ and $(\mathrm{n}, \mathrm{p})$ reaction on ${ }^{35} \mathrm{Cl}$. The thermal absorption on ${ }^{6} \mathrm{Li}$ results in a signal of

${ }^{*}$ Corresponding author.

This is an Open Access article published by World Scientific Publishing Company. It is distributed under the terms of the Creative Commons Attribution 4.0 (CC-BY) License. Further distribution of this work is permitted, provided the original work is properly cited. 
about $3.2 \mathrm{MeV}_{\text {ee}}$; the signal due to $(\mathrm{n}, \mathrm{p})^{35} \mathrm{Cl}$ lies below $3.2 \mathrm{MeV}_{\text {ee, }}$ up to a neutron energy of about $2.5 \mathrm{MeV} .{ }^{5}$ For further discrimination between gamma-ray and neutroninduced events, processing of the signal by pulse-shape discrimination (PSD) is possible.

In this paper, we report on determining the detector-response function to gamma rays through measurements with calibrated gamma-ray sources, carried out in a well-defined, reproducible geometry. The measurement data allowed us to characterize our CLYC detector in terms of energy resolution and full energy efficiency as a function of gammaray energy. As part of this characterization, we developed a Monte Carlo model of the CLYC detector used with the codes $\mathrm{MCNP}^{6}$ and $\mathrm{MCNP}^{6} \mathrm{CP}^{7}$ to determine the detector's energy response. The model was benchmarked with the measurements above.

\section{Detector Response Function}

We express the measured pulse-height spectrum $N\left(E_{d}\right)$ of a radiation detector to an irradiating $\gamma$-ray fluence spectrum $\Phi_{E}$ as

$$
N\left(E_{d}\right)=\iiint R_{2}\left(E_{d}, E_{e}\right) R_{1}\left(E_{e}, E_{\gamma}, \vec{r}\right) \Phi_{E}\left(E_{\gamma}, \vec{r}\right) d E_{e} d E_{\gamma} d \vec{r},
$$

where $E_{d}$ is the observed signal and $E_{\gamma}$ the gamma-ray energy, $R_{l}\left(E_{e}, E_{\gamma}\right)$ describes the transfer of gamma-ray energy $E_{\gamma}$ in the electron energy $E_{e}$, and $R_{2}\left(E_{d}, E_{e}\right)$ relates the electron energy to $E_{d}$. The vector $\vec{r}$ defines the position, and the corresponding integration runs over the detector volume.

The response $R_{l}$ is determined by the gamma-ray transport in the detector material and may be calculated by Monte Carlo simulations, as may the term $\Phi_{E}$. The function $R_{2}$ describing the transfer of electron energy into the observed signal depends on processes such as ionization, scintillation, photomultiplication, and signal processing, which are too complex to be modeled or described in detail. In a first approximation, this transfer can be represented by a Gaussian function,

$$
R_{2}\left(E_{d}, \mu\left(E_{e}\right)\right)=\frac{1}{\sqrt{2 \pi \sigma_{\mu}}} \exp \left[-\frac{\left(E_{d}-\mu\left(E_{e}\right)\right)^{2}}{2 \sigma_{\mu}^{2}}\right],
$$

which also accounts for statistical fluctuations in observed energy. The conversion of the energy of the electron $E_{e}$ into the observed signal is defined by the relationship $\mu\left(E_{e}\right)$ and a resolution broadening $\sigma_{\mu}$, which are functional forms of $E_{e}$ and $\mu\left(E_{e}\right)$, respectively. In an ideal case, $\mu\left(E_{e}\right)$ and $\sigma_{\mu}{ }^{2}$ are directly proportional to energy $E_{e}$. The determination of these functions, called the energy-calibration and resolution functions, respectively, can only be determined phenomenologically — an objective of this work. 


\section{Experiments}

\subsection{Equipment}

We employed a CLYC detector from Scionix, ${ }^{8}$ consisting of a cylindrical $2.54 \mathrm{~cm} \times$ $2.54 \mathrm{~cm}$ CLYC crystal and a Hamamatsu R13089-100 photomultiplier (PMT) mounted through a borosilicate glass window. The enrichment in ${ }^{6} \mathrm{Li}$ was $96 \%$.

A CAEN DT1470ET high-voltage unit was used to supply a $-1200 \mathrm{~V}_{\mathrm{DC}}$ to the PMT. The positive signal from the ninth dynode of the PMT was sent to an Ortec 113 model preamplifier with a 200-pF input capacitance, while the anode was terminated with a 50Ohm resistance. The preamplified signal was then sent to a MCA527 unit from GBSElektronik. ${ }^{9}$ The signal was processed with a trigger filter $(+1,0,-2,0,+1)$, with pileup rejection mode and baseline restoring at $1 / 16$. The shaping time was set to $0.5 \mu$ s and the flat-top time to $5 \mu \mathrm{s}$. The gain was about $1 \mathrm{keV} / \mathrm{ch}$. Pole-zero compensation was verified before each measurement. The detector response was measured over 2048 channels.

The background was monitored regularly during the measurements and found stable. The measured spectra were corrected for dead time and background before use in data analysis.

\subsection{Sources}

Experiments in reproducible geometry were carried out using calibrated point gamma-ray sources. The sources used to measure the detector response ranged from $60 \mathrm{keV}$ to 1.3 MeV, as indicated in Table 1 in Sec. 4.

\subsection{Setup}

The sources were placed above a Plexiglas holder with concentric circles that allow accurate positioning at the center, as shown in Fig.1. This setup allowed changes in the positions of the source and detector. Measurements were carried out at three distances between the detector surface and source: $0.67 \mathrm{~cm}, 4.73 \mathrm{~cm}$, and $10.75 \mathrm{~cm}$.

Repetitive measurements with a ${ }^{137} \mathrm{Cs}$ source were carried out at $4.73 \mathrm{~cm}$ distance. These repetitive measurements allowed us to assess the effect of source positioning on detector response.

\section{Modeling and Monte Carlo Simulations}

We developed a Monte Carlo model of the measurement setup, accounting for geometry and materials as accurately as possible. The information previously discussed concerning the CLYC was complemented with additional information from Scionix. We modeled the detector and materials around the detector that influenced the response. The crystal was covered with PTFE reflector and surrounded by an aluminium casing. A $\mu$-metal shield covered the PMT to provide electromagnetic insulation, and design information was verified by means of x-ray photography. Careful analysis revealed that the 
cylindrical-crystal length and diameter were $2.44 \mathrm{~cm}$ and $2.46 \mathrm{~cm}$, respectively, slightly smaller than the nominal values of $2.54 \mathrm{~cm}$.

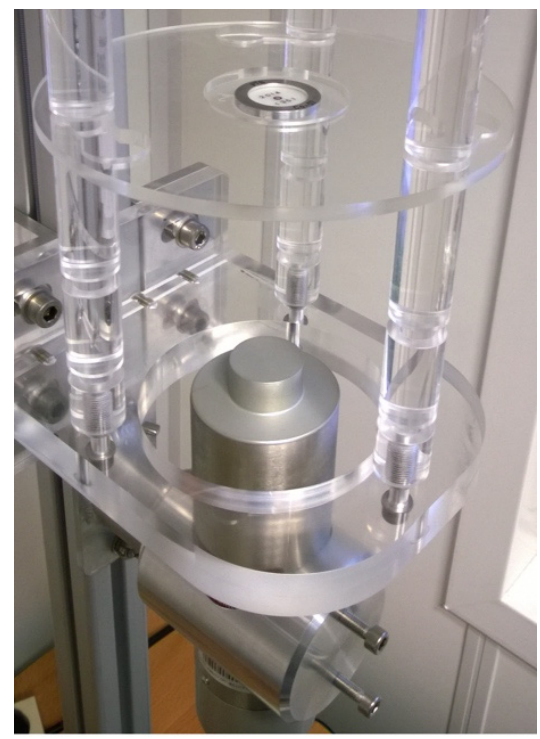

Fig. 1. The measurement setup.

Previous work on the modeling of CLYC detector response can be found in Refs. 10, 11 and 12, which study detector responses to neutrons. In this work, we focused on determining the unbroadened response to gamma-ray sources by means of the F8 tally (energy deposition).

Monte Carlo codes MCNP-CP ${ }^{7}$ and $\mathrm{MCNP}^{6}$ were used to simulate the response of the detection system. In standard versions of the MCNP code, the detector response to mono-energetic gamma rays can be determined. MCNP-CP is a patch for standard MCNP Version $4 \mathrm{c} \operatorname{code}^{13}$ that allows simulations of a source of correlated nuclear particles. MCNP-CP performs a statistical simulation of processes coming with the radioactive decay of a specified radionuclide, yielding characteristics of emitted correlated nuclear particles, which are then tracked through the problem geometry within the same history. In this way, we account for the quasi-simultaneous emission of more than one gamma ray following radioactive decay, thereby accounting for phenomena such as coincidence peaks when more than one gamma ray is emitted. Variance reduction techniques cannot be used with MCNP-CP. The pulse height F8 tally was used to determine the $R_{I} \Phi_{E}$ integrated over the detector volume and $E_{\gamma}$ in Eq. (1).

It is expected that the difference between MCNP6 and MCNP-CP vanishes as the distance between the source and detector increases. At short distances, effects related to coincidence summing effects are more evident. This interpretation is confirmed by comparing the full-energy efficiency obtained with the two codes for the energies 
indicated in Table 1. Since the tally data of MCNP-CP are normalized per decay and those of MCNP6 per gamma ray, we accounted for the emission probabilities of the gamma rays that were used by MCNP-CP.

Table 1. Change in full-energy efficiency obtained with MCNP6 and MCNP$\mathrm{CP}$ for different sources as a function of the distance $d$ between detector and source.

\begin{tabular}{ccccc}
\hline Main $\mathrm{E}_{\gamma} / \mathrm{keV}$ & Radionuclide & $\mathrm{d}=0.67 \mathrm{~cm}$ & $\mathrm{~d}=4.73 \mathrm{~cm}$ & $\mathrm{~d}=10.75 \mathrm{~cm}$ \\
\hline 59.541 & ${ }^{241} \mathrm{Am}$ & $-0.09 \pm 0.10$ & $0.09 \pm 0.11$ & $0.19 \pm 0.28$ \\
88.034 & ${ }^{109} \mathrm{Cd}$ & $0.06 \pm 0.08$ & $0.06 \pm 0.10$ & $-0.45 \pm 0.28$ \\
122.061 & ${ }^{57} \mathrm{Co}$ & $0.33 \pm 0.15$ & $0.09 \pm 0.17$ & $-0.26 \pm 0.23$ \\
165.857 & ${ }^{139} \mathrm{Ce}$ & $12.51 \pm 0.17$ & $0.85 \pm 0.17$ & $-0.03 \pm 0.25$ \\
391.698 & ${ }^{113} \mathrm{Sn}$ & $0.01 \pm 0.16$ & $0.43 \pm 0.14$ & $0.49 \pm 0.24$ \\
514.007 & ${ }^{85} \mathrm{Sr}$ & $5.15 \pm 0.20$ & $5.11 \pm 0.18$ & $4.58 \pm 0.23$ \\
661.657 & ${ }^{137} \mathrm{Cs}$ & $-0.08 \pm 0.19$ & $0.81 \pm 0.18$ & $0.72 \pm 0.25$ \\
834.848 & ${ }^{54} \mathrm{Mn}$ & $0.83 \pm 0.21$ & $1.07 \pm 0.18$ & $1.13 \pm 0.26$ \\
1115.539 & ${ }^{65} \mathrm{Zn}$ & $0.92 \pm 0.19$ & $1.56 \pm 0.18$ & $1.41 \pm 0.34$ \\
1274.530 & ${ }^{22} \mathrm{Na}$ & $5.12 \pm 0.20$ & $2.19 \pm 0.18$ & $1.39 \pm 0.36$ \\
1332.492 & ${ }^{60} \mathrm{Co}$ & $4.56 \pm 0.21$ & $2.27 \pm 0.18$ & $1.97 \pm 0.31$ \\
\hline
\end{tabular}

The data in Table 1 reveal that the differences between MCNP6 and MCNP-CP diminish as the distance between the source and detector increases, for all sources except ${ }^{85} \mathrm{Sr}$. Note that for ${ }^{85} \mathrm{Sr}$, a systematic difference of about $5 \%$ between MCNP6 and MCNP-CP is observed at all distances. Investigation showed that this systematic effect can be related to the ENSDF2 data used by MCNP-CP to simulate ${ }^{85} \mathrm{Sr}$ decay.

\section{Data Analysis}

To determine the energy-calibration function $\mu$ and resolution function $\sigma_{\mu}$, a procedure similar to that in Refs. 14 and 15 is applied. The measured response $N_{\exp }$ is compared to the theoretical $N_{f i t}$ using a weighted least-square fit procedure. The detector response $N_{f i t}$,

$$
N_{f i t}\left(E_{d}\right)=\mathrm{C} \iiint R_{2}\left(E_{d}, E_{e}\right) R_{1}\left(E_{e}, E_{\gamma}, \vec{r}\right) \Phi_{E}\left(E_{\gamma}, \vec{r}\right) d E_{e} d E_{\gamma} d \vec{r}
$$

is calculated based on the results of the Monte Carlo simulations, so that the only unknowns are the normalization factor $C$ and the parameters of the functional forms $\mu$ and $\sigma_{\mu}$ of the Gaussian function in Eq. (2).

To determine $C, \mu$, and $\sigma_{\mu}$ for a particular $E_{e}$ from the measured spectrum, it is sufficient to fit the measured spectrum in the peak region. The free parameters $\alpha$ and $\beta$, which link the observed amplitude and resolution by the expressions

$$
\mu=\alpha E_{e}
$$


and

$$
\sigma_{\mu}^{2}=\beta \mu,
$$

together with the normalization factor $C$, can be obtained with the weighted least-square fit procedure by minimizing the expression

$$
\chi^{2}=\sum_{i=1}^{n} \frac{\left(N_{\mathrm{exp}, i}-N_{f i t, i}\right)^{2}}{s_{N, i}^{2}}
$$

where $\mathrm{s}_{N}$ is the uncertainty on the measured spectrum.

To implement the outlined procedure, a Root ${ }^{16}$-based code was developed to allow direct comparison between experimental and simulated data by convoluting the unbroadened spectra obtained from MCNP with the detector-resolution function, rebinning the convoluted data from energy to channel, and scaling the experimental data to the simulated spectra with a normalization constant.

This procedure was repeated for all measured gamma-ray sources, and the parameters $\alpha, \beta$, and $\mathrm{C}$ were determined.

\section{Results}

\subsection{Repetitive measurements}

We carried out a statistical analysis of nine repetitive measurements with a ${ }^{137} \mathrm{Cs}$ source at a $4.73 \mathrm{~cm}$ distance. The distance remained constant, while the source was removed and repositioned every time. Each measurement was analysed and the parameters $C, \mu$, and $\sigma_{\mu}$ were determined with their statistical uncertainty. To study the presence of other uncertainty components, a $\chi^{2}$ analysis was performed on the results of the repetitive measurements by evaluating the quantity

$$
\chi^{2}=\sum_{i=1}^{n} \frac{\left(\theta_{i}-\bar{\theta}_{i}\right)^{2}}{s_{\theta, i}^{2}},
$$

where $\theta_{i}$ and $s \theta_{, i}$ are the parameters and its uncertainty, respectively, as deduced from the fit of each measurement cycle $i$. The quantity $\bar{\theta}_{i}$ is the average value deduced from $n$ repetitive measurements. From the $\chi^{2} / v$ per degree of freedom resulting from repetitive measurements with good counting statistics, the overall additional uncertainty was estimated and is reported in Table 2. For a single measurement, the total uncertainty should be obtained by combining statistical uncertainty with this uncertainty component.

The results reveal that another component dominates the parameters of the energy calibration; this is probably related to the stability of high voltage or PMT response, or both. The uncertainty on the resolution broadening is well described and does not require additional components. The positioning of the source has an effect comparable with that associated with counting statistics on the parameter $\mathrm{C}$. 
Table 2. Chi-square analysis of repetitive measurements and uncertainty components.

\begin{tabular}{llll}
\hline & $\mathrm{C}$ & $\mu$ & $\sigma_{\mu}$ \\
\hline $100 \times \mathrm{s}_{\theta, i} / \theta_{\mathrm{i}}$ (statistics) & 0.5 & 0.01 & 1.3 \\
$\chi^{2} / \nu$ & 1.8 & 375 & 1.1 \\
$100 \times \mathrm{s}_{\theta, i} / \theta_{\mathrm{i}}$ (other) & 0.4 & 0.15 & 0.5 \\
\hline
\end{tabular}

\subsection{Energy dependence}

The behaviour of $\alpha$ and $\beta$ was studied as a function of $E_{\gamma}$. Studying the values of the $\alpha$ parameter over the measurement campaign, which lasted more than a month, no clear trend with energy is visible, and the data have an average value of $0.999 \mathrm{ch} / \mathrm{keV}$ with a standard deviation of $0.7 \%$. This value is larger than the value of $0.15 \%$ given in Table 2 and may be due to the long-term stability of high voltage, PMT, or electronics. Our results suggest that the behavior of the system is linear and the presence of an offset cannot be proven.

A trend with energy can be observed for the $\beta / \alpha$ parameter, as shown in Fig. 2. At $661.7 \mathrm{keV}$, the corresponding full width at half maximum (FWHM) is $5.7 \%$. The fact that $\beta / \alpha$ is not constant indicates that the energy-deposition process is not based on statistical processes only. The observed energy dependence indicates that other factors affect energy resolution, such as local fluctuations in scintillation efficiency, less-than-perfect light reflection at the crystal surface, effects introduced by the PMT, such as the uniformity of the photoelectron collection, and statistical fluctuation in the electron multiplication, as explained in Ref. 17.
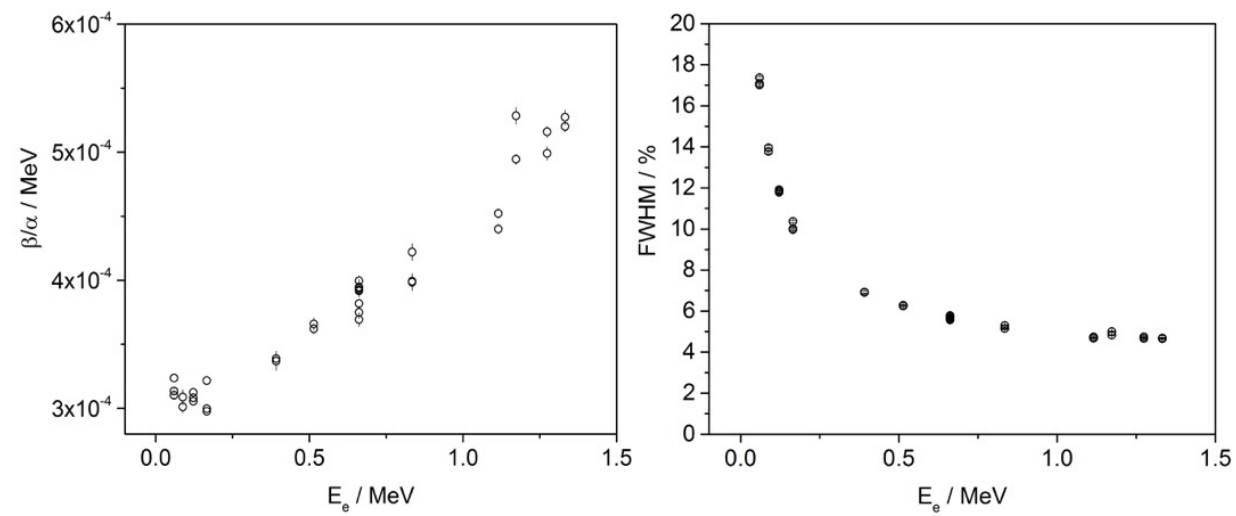

Fig. 2. The parameter $\beta / \alpha$ and corresponding FWHM as a function of the electron energy $\mathrm{E}_{\mathrm{e}}$.

In addition to $\mu$ and $\sigma_{\mu}$ parameters, the quantity $\mathrm{C}$ in Eq. (3) was also determined: if the response $R_{I}$ is expressed per decayed nuclei and $N_{\text {exp }, i}$ are the background corrected 
counts within the measurement time, then the quantity $C$ represents the number of nuclei decayed during the measurement. By comparing $C$ with the calculated number of nuclei based on the certificate information, we can assess the accuracy of the model in absolute terms. The associated full-energy peak efficiency can then be determined by combining the results of this comparison and the efficiency obtained with MCNP-CP.

The results obtained for the $4.73 \mathrm{~cm}$ distance are shown in Fig. 3. The ratio between nominal and model calculated number of decayed nuclei reveals a trend with energy, which is also observed at the other distances.
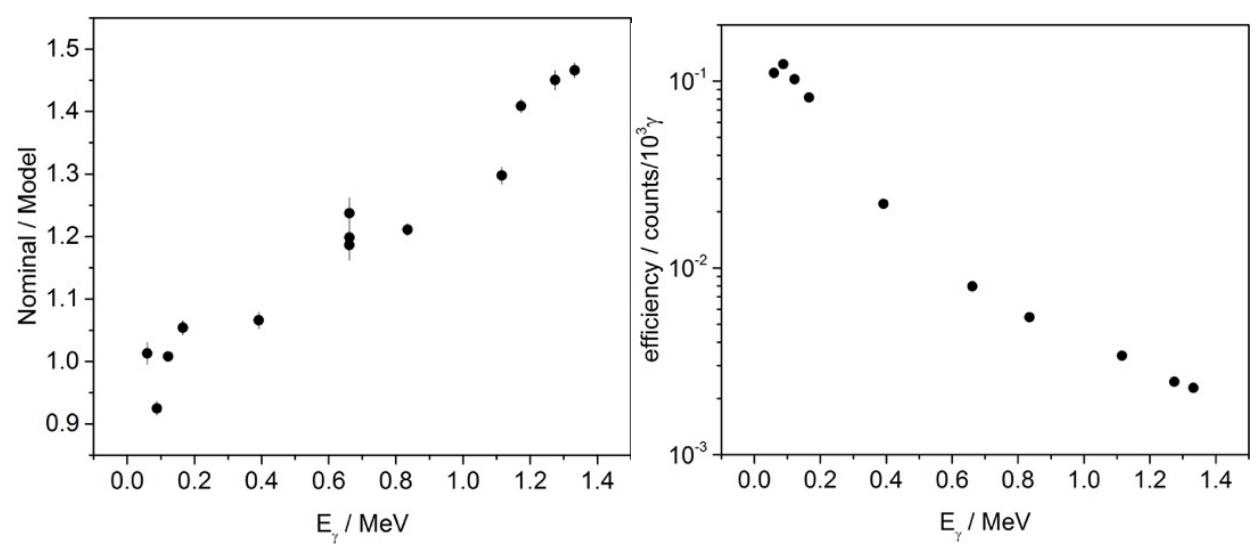

Fig. 3. Ratio between nominal and model calculated number of decayed nuclei for each measurement as a function of gamma-ray energy (left). Experimental full-energy peak efficiency at $4.73 \mathrm{~cm}$ distance (right).

\subsection{Design variations}

The obtained data reveal that the calculated response is overestimated. To understand the observed discrepancy, we carried out additional simulations to identify which model parameters most affect full-energy peak efficiency. The MCNP6 simulations were carried out at $4.73 \mathrm{~cm}$ under various parameters and compared to the reference case. Only one parameter at a time was varied.

The following parameters that affect response were changed:

- Distance of source detector (increased by $1 \mathrm{~cm}$ )

- CLYC crystal density (reduced by $10 \%$ )

- Diameter of CLYC crystal (reduced by $1.4 \mathrm{~mm}$ )

- Length of CLYC crystal (reduced by $2.4 \mathrm{~mm}$ ), while keeping the same distance for the source detector.

The corresponding changes in full-energy peak efficiency are given in Fig. 4.

A change in distance results in a systematic change in efficiency, almost independent of gamma-ray energy. At low energy, the change is higher because the mean free path is shorter, and therefore a change in distance has a greater effect on the response. Similarly, a change in crystal diameter shows very little dependence on gamma-ray energy. 
Changes in detector length and crystal density show more evident energy dependence and are consistent with the observed trend.

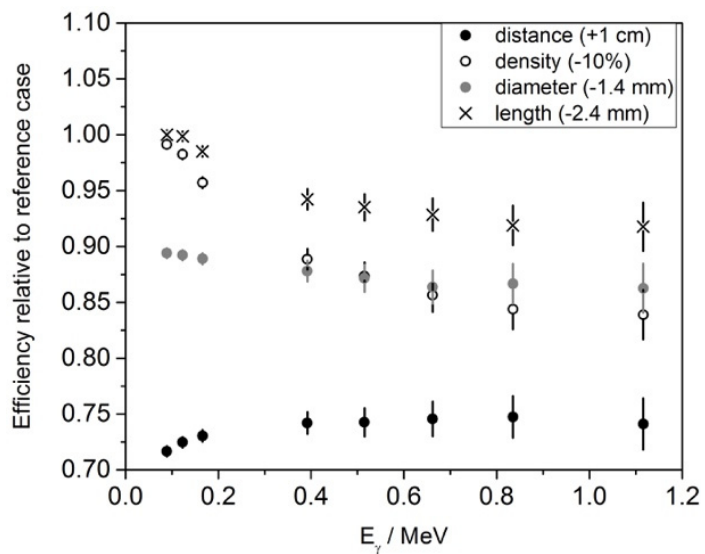

Fig. 4. Change in full-energy peak efficiency for different design variations.

The distance was accurately measured and is a parameter that could well be reproduced within less than $1 \mathrm{~mm}$. The crystal diameter and length were verified by x-ray photography, which revealed that the crystal was slightly small compared to nominal values. This difference was taken into account in the reference calculations, where we used a length of $2.44 \mathrm{~cm}$ and a radiographic measurement of $2.46 \mathrm{~cm}$, while nominal values of $2.54 \mathrm{~cm}$ were given by the manufacturer.

The density of $3.31 \mathrm{~g} / \mathrm{cm}^{3}$ is a parameter that we could not verify, and was taken from Ref. 18. The trend in Fig. 4 is consistent with the results in Fig. 3.

\section{Conclusions}

This paper presents a method to characterize a CLYC detector in terms of detector response to gamma ray, linearity, and energy resolution. Measurements with calibrated gamma-ray point-sources with an analog measuring chain were employed in a wellgiven, reproducible geometry. The detector was modeled and detector responses obtained with Monte Carlo MCNP-CP were used in the data analysis. Experiments and simulations were compared absolutely, revealing a discrepancy between model and experiments that became evident above $400 \mathrm{keV}$. Possible design variations were investigated as a source of this discrepancy. Differences in crystal density best explain the observed discrepancy.

Future work will include testing the performance of the detector with neutron sources and data acquisition based on a CAEN 5730B digitizer. This system allows acquisition of the signal waveform of the photomultiplier tube with a 2 ns resolution and can perform pulse-shape discrimination on board. 


\section{References}

1. C. Combes, P. Dorenbos, C. v. Eijk, K. Kramer and H. Gudel, J. Luminescence 182, 299 (1999).

2. N. D’Olympia, P. Chowdhury, C. Lister, J. Glodo, R. Hawrami, K. Shah and U. Shirwadkar, Nucl. Instr. Meth. Phys. Res. 714, 121 (2013).

3. R. Machrafi, N. Khan and A. Miller, Rad. Meas. 70, 5 (2014).

4. A. Giaz, Nucl. Instr. Meth. A 1810, 132 (2016).

5. N. D’Olympia, P. Chowdhury, C.J. Guess, T. Harrington, E.G. Jackson, S. Lakshmi, C.J. Lister, J. Glodo, R. Hawrami, K. Shah and U. Shirwadkar, Nucl. Instr. Meth. A 694, 140 (2012).

6. MCNP6 Users Manual - Code Version 6.1.1 beta, LA-CP-14-00745, June 2014.

7. A. N. Berlizov, MCNP-CP Upgrade Patch Version 3.2, A Correlated Particle Source Extension Of A General Purpose Monte Carlo N-Particle Transport Code Manual, May 2009.

8. http://scionix.nl/

9. MCA-527, Digital Multi-Channel Analyzer, GBS-Elektronic, User Manual (2012)

10. M. M. Bourne, C. Mussi, E. C.; Miller, S. D. Clarke, S. A. Pozzi, A. Gueorguiev, Nucl. Instr. Meth. Phys. Res. A 736, 124 (2014).

11. N. D’Olympia, P. Chowdhury, E. G. Jackson, C. Lister, Nucl. Instr. Meth. A 763, 433 (2014).

12. R. Machrafi, A. L. Miller, N. Khan, Rad. Meas. 80, 10 (2015).

13. J. F. Briesmeister, MCNP - A general Monte Carlo N-particle transport code, Los Alamos National Laboratory Report, 1997, LA-12625-M.

14. M. Weyrauch, A. Casnati, P. Schillebeeckx and M. Clapham, Nucl. Instr. Meth. A 405, 442 (1998).

15. A. Borella, G. Aerts, F. Gunsing, M. Moxon, P. Schillebeeckx and R. Wynants, Nucl. Instr. Meth. A, 577626 (2007).

16. R. Brun and F. Rademakers, Nucl. Instr. Meth. A 389, 81 (1997).

17. G. F. Knoll, in Radiation Detection and Measurement, 2nd Edition, ISBN 13: 9780471815044 (Wiley, 1989), pp. 312-314.

18. http://rmdinc.com/clyc/. 